\title{
Hemorheological properties in patients with type-1 and type-2 diabetes mellitus
}

\author{
Irina A. Sokolova ${ }^{1}$, Aleksandrina V. Kachalova ${ }^{1,2}$, Anastasiya A. Fabrichnova ${ }^{2}$, Yulia A. Kovaleva ${ }^{3}$, \\ Aleksandr A. Shahnazarov ${ }^{1}$, and Vladimir B. Koshelev ${ }^{2 *}$ \\ ${ }^{1}$ Laboratory of Biomechanics, Institute of Mechanics, M.V. Lomonosov Moscow State University, Michurinsky pr., 1 , \\ Moscow 119192, Russia \\ 2 Faculty of Fundamental Medicine, M.V. Lomonosov Moscow State University, Lomonosovsky pr., 27-1, \\ Moscow 119991, Russia \\ ${ }^{3}$ Department of Therapeutic Endocrinology, M.F. Vladimirsky Moscow Regional Research and Clinical Institute, \\ Shepkina str., 61/2, Moscow129110, Russia \\ * e-mail: vkoshelev@fbm.msu.ru
}

\begin{abstract}
The hemorheologic disorders, which are potentially deleterious in a wide range of pathologies, were studied in patients with type- 1 and type- 2 diabetes mellitus. The significant determinants of blood viscosity, red blood cells (RBC) deformability and RBC reversible aggregation were assessed by ektacytometry and by laser backscattering technique, respectively. For both types of the disease, we observed acceleration of the first phase of RBC aggregation that contrasted with its deceleration at the second phase. The hydrodynamic stability of the aggregates, especially the smallest ones, exceeded the normal values in both cases. The influence of GP IIb/IIIa receptor inhibitor, monafram on RBC aggregation and disaggregation was the same in the cases of patients and normal subjects. The maximal shear induced RBC stretching exceeded the normal one in patients of both groups. The data reveal not only pathological but also compensatory character of hemorheological alterations in patients with type-1 and type-2 diabetes mellitus. (C) 2017 Journal of Biomedical Photonics \& Engineering.
\end{abstract}

Keywords: diabetes mellitus; RBC aggregation; RBC deformability; monafram.

Paper \#3219 received 16 Jan 2017; revised manuscript received 15 Feb 2017; accepted for publication 15 Jun 2017; published online 13 Jul 2017. doi: 10.18287/JBPE17.03.030402. [Special Issue. Years in Biophotonics: 70th Anniversary of Prof. A.V. Priezzhev].

\section{References}

1. H. J. Meiselman, "Red blood cell aggregation: 45 years being curious," Biorheology 46(1), 1-19 (2009).

2. J. Radosinska, and N. Vrbjar, "The role of red blood cell deformability and Na,K-ATPase function in selected risk factors of cardiovascular diseases in humans: focus on hypertension, diabetes mellitus and hypercholesterolemia," Physiol. Res. 65(Suppl. 1), S43-S54 (2016).

3. S. Mendis, S. Davis, and B. Norrving, "Organizational Update: The World Health Organization Global Status Report on Noncommunicable Diseases 2014; One More Landmark Step in the Combat Against Stroke and Vascular Disease," Stroke 46, e121-e122 (2015).

4. P. Vague, and I. Juhan, "Red cell deformability, platelet aggregation, and insulin action," Diabetes 32(Suppl. 2), 88-91 (1983).

5. C. Lacombe, C. Bucherer, and J. C. Lelievre, "Hemorheological improvement after Daflon 500 mg treatment in diabetes," Int. Angiol. 7(Suppl. 2), 21-24 (1988).

6. M. Cho, S. Shin, H. M. Kwon, H. Chung, and B. K. Lee, "Effect of clinical and RBC hemorheological parameters on myocardial perfusion in patients with type 2 diabetes mellitus," Biorheology 51(2-3), 215-226 (2014).

7. N. Pargalava, M. Mantskava, and G. Mchedlishvili, "Regional and systemic hemorheological disorders during feet diabetic gangrene," Clin. Hemorheol. Microcirc. 30(3-4), 457-459 (2004). 
8. G. Cicco, and S. Cicco, "Hemorheology and microcirculation in some pathologies of internal medicine," Minerva Med. 98(6), 625-631 (2007) [in Italian].

9. S. Chien, and K.-M. Jan, "Ultrastructure basis of the mechanism of rouleaux formation," Microvascular Research 5, 155-166 (1973).

10. D. Lominadze, and W. Dean, "Involvement of fibrinogen specific binding in erythrocyte aggregation," FEBS Lett. 513(1-3), 41-44 (2002).

11. I. A. Sokolova, A. V. Muravyov, M. D. Khokhlova, S. Y. Rikova, E. V. Lyubin, M. A. Gafarova, M. N. Skryabina, A. A. Fedyanin, D. V. Kryukova, and A. A. Shahnazarov, "An effect of glycoprotein IIb/IIIa inhibitors on the kinetics of red blood cells aggregation," Clin. Hemorheol. Microcirc. 57(3), 291-302 (2014).

12. E. Straface, R. Rivabene, R. Masella, M. Santulli, R. Paganelli, and W. Malorni, "Structural changes of the erythrocyte as a marker of non-insulin-dependent diabetes: protective effects of N-acetylcysteine," Biochem. Biophys. Res. Commun 290(5), 1393-1398 (2002).

13. M. Donner, M. Siadat, and J. F. Stoltz, "Erythrocyte aggregation: approach by light scattering determination," Biorheology 25(1-2), 367-375 (1988).

14. M. Bessis, and N. Mohandas, "A diffractometric method for the measurement of cellular deformability," Blood Cells 8(3), 307-313 (1975).

15. T. V. Korotaeva, N. N. Firsov, A. Bjelle, and M. A. Vishlova, "Erythrocytes aggregation in healthy donors at native and standard hematocrit: the influence of sex, age, immunoglobulins and fibrinogen concentrations. Standardization of parameters," Clin. Hemorheol. Microcirc. 36(4), 335-343 (2007).

16. N. N. Firsov, N. V. Klimova, A. V. Priezzhev, and A. Yu. Tyurina, "Fundamental laws of the deformational behaviour of erythrocytes in shear flow," Journal of Engineering Physics and Thermophysics 79(1), 118-124 (2006) [in Russian].

17. O. V. Filatova, A. A. Sidorenko, and S. A. Agarkova, "Effects of age and sex on rheological properties of blood," Human Physiology 41(4), 437-443 (2015) [in Russian].

18. M. Mantskava, N. Momtselidze, N. Pargalava, and G. Mchedlishvili, "Hemorheological disorders in patients with type 1 or 2 diabetes mellitus and foot gangrene," Clin. Hemorheol. Microcirc. 35(1-2), 307-310 (2006).

19. I. Sokolova, M. Gafarova, M. Khokhlova, A. Muravyev, E. Lyubin, M. Skryabina, A. Fedyanin, T. Krasnova, and A. Shahnazarov, "Glycoprotein IIB-IIIA inhibitor, monafram decelerate the early phase of red blood cells aggregation," Journal of Cellular Biotechnology 2(1), 15-22 (2016).

20. W. Subasinghe, and D. M. Spence, "Simultaneous determination of cell aging and ATP release from erythrocytes and its implications in type 2 diabetes," Anal. Chim. Acta. 618(2), 227-233 (2008).

\section{Introduction}

The abnormalities of biophysical properties of blood are often related to enhanced $\mathrm{RBC}$ rigidity, which results in microcirculatory disturbances and aggravation effects in different diseases $[1,2]$. One of these diseases is the diabetes mellitus (DM) that now has the character of a non-infection epidemy because of its prevalence [3]. $\mathrm{DM}$ is characterised by the absolute or relative deficiency of insulin, leading to hyperglycaemia, and long ago it was revealed that DM can accompanied by the decreased RBC deformability and enhanced RBC aggregation $[4,5]$. These hemorheological disturbances can give rise to ischemic-related tissue damage and stimulate the development of different complications [6]. At the same time, the role of hemorheological pathology in DM is not undisputable: some researchers noted its presence mainly in local regions or did not observe it at all $[7,8]$. The mechanisms of enhanced RBC aggregation are also still unclear. Whatever the explanation of this phenomenon is (the non-specific interaction of biopolymer molecules, mainly fibrinogen, with cell membranes and, hence, the RBC linking by polymer "bridges" [9], or the relative reduction of the macromolecules concentration near the cells surface, giving rise to attractive osmotic forces [1]), the mechanisms of RBC aggregation are mainly considered to be non-specific. Nevertheless, the specific fibrinogen receptors were revealed at RBC membrane [10], and it is possible that, in pathologic cases, this specific fibrinogen-RBC interaction can enhance the stability of $\mathrm{RBC}$ aggregates [11]. In patients with DM, the RBCs were shown to possess some specific features [12], but their possible receptor interaction with fibrinogen (which, in contrast to non-specific coupling, could be subject to pharmacological intervention) remains practically unstudied.

The objective of this study was to investigate RBC deformability and aggregation in patients with diabetes mellitus and, in addition, we aimed to evaluate a possibility of specific fibrinogen binding by RBC membrane as one of the factors which contribute to $\mathrm{RBC}$ aggregation in this disease.

\section{Materials and methods}

\subsection{Subjects}

Blood samples were collected from patients with type-1 or type-2 diabetes mellitus (DM1 and DM2, respectively) and from healthy volunteers of comparable sex and age. The patients were hospitalized in the Department of Therapeutic Endocrinology of the M.F 
Vladimirsky Moscow Regional Research and Clinical Institute (MONIKI), where they passed the standard clinical and laboratory examination. The study was carried out in accordance with the protocols, approved by the Ethics Committee of the MONIKI and FFM MSU, after the receipt of informed consent from all the donors.

The blood samples were withdrawn from the elbow median vein (v. mediana cubiti) and stabilized with ethylene diamine tetraacetate (EDTA, $2 \mathrm{mg} / \mathrm{ml}$ ). They were tested within 2 hours after the sampling.

\subsection{Hemorheological analysis}

The kinetics of RBC aggregation was studied using the light backscattering method [13], and the RBC deformability was tested by means of the ectacytometry method [14] using the coaxial cylindrical aggregometerdeformometer LADE (the rheological gap $0.9 \mathrm{~mm}$, $\lambda=650 \mathrm{~nm}$, the laser radiation power $1 \mathrm{~mW}$, ReoMedLab, Ltd., Russia) at the temperature $25^{\circ} \mathrm{C}$. The cells reversible aggregation was studied under the hematocrit (Hct) $\sim 0.40$ that was standardized by correcting the Hct of the whole blood. In the course of the experiment after a short period of high-velocity flow, the flow was stopped and the decrease of the backscattered intensity (I) as a function of the time (T) was observed during 2 minutes, reflecting the process of spontaneous RBC aggregation. Then at the effective shear velocity $\dot{\gamma}$ varying from $\sim 2.5 \mathrm{~s}^{-1}$ to $\sim 130 \mathrm{~s}^{-1}$ the hydrodynamic stability of RBC aggregates was tested. The dependences $\mathrm{I}(\mathrm{T})$ and $\mathrm{I}(\dot{\gamma})$ were rectified in semilogarithmic coordinates. The characteristic times, which characterized a duration of the first and the second phase of $\mathrm{RBC}$ aggregation, were calculated using the formula $t_{1,2}=T / \ln$ I. The parameter $A$ defined as the difference between the initial value of I, corresponding to full disaggregation of cells, and the one in the end of the aggregation process, was used to characterize the degree of RBC aggregation as well as the effective size of the aggregates. The indexes of hydrodynamic strength of the bulk of aggregates and the smallest ones were calculated using the formula $\beta_{1,2}=\dot{\gamma} / \ln$ I. Additionally, we used the index of strength for the largest aggregates $\mathrm{I}_{2.5}$, defined as the percentage of aggregates, disaggregated at the minimal value of $\dot{\gamma} \approx 2.5 \mathrm{~s}^{-1}$. The value of $\mathrm{I}_{2.5}$ is taken with a negative sign to make it an indicator of erythrocytes interaction strength rather than that of disaggregation easiness [15]. To characterize RBC deformability we determined the threshold shear velocity $\dot{\gamma}_{b}$, at which the cell deformation process began in the shear flow. Besides that, we used the indicator tg $\alpha$, reflecting the degree of erythrocyte deformation in the accelerating flow ( $\dot{\gamma}$ changed stepwise from 0 to $\sim 2700 \mathrm{~s}^{-1}$ ). This indicator characterized a slope of the straight line $\operatorname{ID}(\ln \dot{\gamma})$, where ID was the deformability index, i.e., the $\dot{\gamma}$-dependent difference between the maximal and minimal diameter of the light ellipse, occurring as a result of the diffraction of laser beam by the cells and corresponding to the shape of an averaged erythrocyte, divided by the sum of these diameters. The maximal elongation of the cells was characterized by IDmax, determined at the maximal $\dot{\gamma} \approx 2700 \mathrm{~s}-1$ [16].

\subsection{Modification of fibrinogen-RBC binding}

The receptor binding of fibrinogen was affected by monafram, the inhibitor of the glycoprotein GP IIb/IIIa receptors, produced in the Russian Cardiology Research and Production Complex of Moscow. Since under the conditions of our experiment, no practical activation of platelets occurred and the inhibitor affected mainly the erythrocyte receptors [11], we used the samples of whole blood, divided into two parts. To one part (the control one) we added saline (10 $\mu 1$ per $1 \mathrm{ml}$ of blood), and the second one was incubated with monafram $(20 \mathrm{mg} / \mathrm{ml})$ during $30 \mathrm{~min}$. Both portions were tested in random order.

\subsection{Miscellaneous techniques}

The hematocrit was determined by means of microcentrifuging (7000 turns/min., 6 min.; C-702, Apel, Japan). The viscosity of blood plasma was tested by means of the capillary viscometer (the inner diameter of the vertical capillary $0.6 \mathrm{~mm}$, the length $120 \mathrm{~mm}$; ReoMedLab Ltd., Russia). The shape of the cells was monitored using the microscope (40×; Biolam M-1; AO LOMO, Russia).

\subsection{Statistical analysis}

The data were statistically processed using the software package SPSS 11.5. The data distribution was assessed by means of the Shapiro-Wilk statistics. The significance of the difference between the data obtained in the patients and in the normal subjects was assessed using the unpaired Student t-criterion or the MannWhitney criterion. To assess the differences between the control data and the data obtained after the incubation of blood with monafram we used the paired Student $t$ criterion or the Wilcoxon criterion. The correlation relation was characterized using the Spearman statistics.

For all statistical comparisons, p-value $<0.05$ was considered statistically significant. The data are presented as mean value \pm standard deviation (SD).

\section{Results and discussion}

The RBCs of the examined patients with the type- 1 or type-2 diabetes mellitus (DM1 and DM2) held the normal shape. Upon the average, no anomalies of hematocrit and blood plasma viscosity were observed in the DM1 and DM2 groups in comparison with the corresponding control groups (N1 and N2). Analogous difference between the groups DM1 and DM2, as well as between the groups $\mathrm{N} 1$ and N2 were also not significant (see Table 1). 
Table 1 General characteristics of patients with the type-1 (DM1) and type-2 (DM2) diabetes mellitus and healthy volunteers of the corresponding control groups ( $\mathrm{N} 1$ and N2), as well as hematocrit (Hct) and blood plasma viscosity $\left(\eta_{\mathrm{pl}}\right)$.

\begin{tabular}{llcc}
\hline & Age (years) and number of people (n) & Hct & $\boldsymbol{\eta}_{\mathbf{p l}}, \mathbf{m P a} \cdot \mathbf{s}$ \\
\hline DM1 & $29.8 \pm 9.4, \mathrm{n}=20$ (7 men and 13 women) & $0.40 \pm 0.04$ & $1.58 \pm 0.17$ \\
\hline N1 & $27.4 \pm 5.5, \mathrm{n}=113(40$ men and 73 women) & $0.41 \pm 0.04$ & $1.55 \pm 0.30$ \\
\hline DM2 & $60.4 \pm 7.6^{\wedge}, \mathrm{n}=15(1$ man and 14 women) & $0.39 \pm 0.04$ & $1.64 \pm 0.19$ \\
\hline N2 & $56.0 \pm 13.8^{\wedge}, \mathrm{n}=11$ (1 man and 10 women) & $0.41 \pm 0.03$ & $1.62 \pm 0.20$ \\
\hline
\end{tabular}

Note: ${ }^{\wedge} \mathrm{p}<0.001$, the comparison of DM1 and DM2, as well as N1 and N2.

Table 2 Characteristic time of the first and the second phase of RBC red aggregation $\left(t_{1}\right.$ and $\left.t_{2}\right)$, the effective size of aggregates (A), and the indexes of hydrodynamic strength for large aggregates $\left(\mathrm{I}_{2.5}\right)$ and aggregates in bulk $\left(\beta_{1}\right)$ in type1 and type-2 diabetes mellitus patients (DM1 and DM2) and in normal subjects, (N1 and N2, respectively).

\begin{tabular}{llllll}
\hline & \multicolumn{1}{c}{$\mathbf{t}_{\mathbf{1}}, \mathbf{s}$} & \multicolumn{1}{c}{$\mathbf{t}_{\mathbf{2}}, \mathbf{s}$} & $\mathbf{A}, \mathbf{r e l}$ units & $\mathbf{I}_{\mathbf{2 . 5}}, \mathbf{\%}$ & $\mathbf{b}_{\mathbf{1}}, \mathbf{s}^{\mathbf{- 1}}$ \\
\hline $\mathrm{DM} 1$ & $7.83 \pm 1.74^{*}$ & $37.4 \pm 3.7^{* *}$ & $1330 \pm 435$ & $-20.1 \pm 4.1^{*}$ & $34.1 \pm 8.8^{* *}$ \\
\hline $\mathrm{N} 1$ & $9.72 \pm 3.60$ & $34.4 \pm 3.7$ & $1239 \pm 502$ & $-23.5 \pm 7.3$ & $28.5 \pm 12.7$ \\
\hline $\mathrm{DM} 2$ & $6.39 \pm 1.27^{\wedge \wedge}$ & $38.6 \pm 3.6^{* *}$ & $1470 \pm 250^{\wedge}$ & $-17.7 \pm 4.7$ & $34.8 \pm 11.2$ \\
\hline $\mathrm{N} 2$ & $7.75 \pm 3.51^{\wedge}$ & $31.3 \pm 9.4$ & $1406 \pm 567$ & $-21.6 \pm 9.9$ & $30.6 \pm 10.1$
\end{tabular}

Note: $* \mathrm{p}<0.01, * * \mathrm{p}<0.005$, the comparison of DM1 and DM2, as well as of N1 and N2.

\subsection{RBC aggregation dynamics in patients with type-1 or type-2 diabetes mellitus}

Both in the presence of the disease and in the normal condition, the known age trends [17] of erythrocyte aggregation enhancement and disaggregation process weakening were found to manifest themselves (compare DM1 and DM2, as well as N1 and N2 in Table 2).

Compared to the corresponding groups of normal subjects, in the case of DM1 and DM2 we observed the signs of "hyperaggregation" (see Table 2) that were more clearly seen in the case of DM1, probably, due to the greater number of members in the appropriate group of the tested patients. The intensification of $\mathrm{RBC}$ aggregation for both DM1 and DM2 was noticed earlier
[18]. However, the detailed analysis of the dynamics of this process revealed an intriguing feature, namely, for both DM1 and DM2, the pathological reduction of $t_{1}$, the time of the first phase of RBC aggregation was accompanied by the prolongation of $t_{2}$, the second phase time (Table 2). The resulting aggregates possessed increased stability, which was explicitly observed in the case of DM1 and as a trend $(\mathrm{p}<0.06)$ in the case of DM2 $\left(I_{2.5}\right.$ and $\beta_{1}$, Table 2$)$. In the normal state, the disaggregation of cells mainly occurred as a single process, and only in $37 \%$ of cases the disintegration of the smallest aggregates was hindered and $\beta_{2}=56.1 \pm 21.8 \mathrm{~s}^{-1}$ was recorded. In the DM1 and DM2 groups, $\beta_{2}$ was recorded in $100 \%$ of the cases,

Table 3 Monafram-related alterations (M) of the characteristic time of the 1-st phase of RBC aggregation $\left(t_{1}\right)$ and the indexes of hydrodynamic strength for largest aggregates $\left(I_{2.5}\right)$ and aggregates in bulk $\left(\beta_{1}\right)$ in the presence of "hyperaggregation" for the type-1 and type-2 diabetes mellitus (DM1h and DM2h) and in the normal state (Nh).

\begin{tabular}{llcll}
\hline & & \multicolumn{1}{c}{$\mathbf{t}_{\mathbf{1}}, \mathbf{c}$} & \multicolumn{1}{c}{$\mathbf{I}_{\mathbf{2} .5}, \mathbf{\%}$} & \multicolumn{1}{c}{$\mathbf{b}_{\mathbf{1}}, \mathbf{s}^{-\mathbf{1}}$} \\
\hline $\mathrm{Nh}$ & control & $8.99 \pm 3.61$ & $-14.5 \pm 5.7$ & $44.0 \pm 10.4$ \\
& $\mathrm{M}$ & $10.06 \pm 3.49^{\&}$ & $-19.2 \pm 7.4^{\& \& \&}$ & $35.3 \pm 10.9^{\& \&}$ \\
\hline DM1h & control & $7.26 \pm 0.90$ & $-17.1 \pm 2.1$ & $40.3 \pm 6.9$ \\
& $\mathrm{M}$ & $7.67 \pm 1.01^{\&}$ & $-19.0 \pm 2.2^{\&}$ & $38.5 \pm 5.8$ \\
\hline DM2h & control & $6.45 \pm 0.75$ & $-17.6 \pm 2.9$ & $47.4 \pm 14.0$ \\
& $\mathrm{M}$ & $7.01 \pm 1.01^{\&}$ & $-19.0 \pm 6.6^{\&}$ & $39.3 \pm 12.6^{\&}$ \\
\hline
\end{tabular}

Note: ${ }^{\&} \mathrm{p}<0.05,{ }^{\& \&} \mathrm{p}<0.01,{ }^{\& \& \&} \mathrm{p}<0.005$, comparison of «M» and «control $»$. 
Table 4 Threshold effective shear velocity, corresponding to the beginning of RBC deformation $\left(\dot{\gamma}_{b}\right)$, as well as the indice of cells deformation degree in the accelerating flow $(\operatorname{tg} \alpha)$ and the index of its maximal deformation (ID max $_{\text {) }}$ in the case of type-1 and type-2 diabetes mellitus (DM1 and DM2) and in the normal state (N1 and N2), respectively.

\begin{tabular}{lccc}
\hline & $\dot{\gamma}_{b}, \mathbf{s}^{-1}$ & tg $\alpha$ & ID $_{\max }$ \\
\hline $\mathrm{DM} 1$ & $30.2 \pm 46.5^{\wedge}$ & $0.0813 \pm 0.0163$ & $0.456 \pm 0.019^{*}$ \\
\hline $\mathrm{N} 1$ & $19.1 \pm 19.6$ & $0.0819 \pm 0.0748$ & $0.426 \pm 0.042$ \\
\hline $\mathrm{DM} 2$ & $12.8 \pm 4.5^{*}$ & $0.0773 \pm 0.0376$ & $0.456 \pm 0.018^{*}$ \\
\hline $\mathrm{N} 2$ & $23.2 \pm 10.5$ & $0.0742 \pm 0.0140$ & $0.399 \pm 0.035$
\end{tabular}

Note: * $\mathrm{p}<0.005$, the comparison of "DM1" and "N1", as well as "DM2" and "N2"; ^ $\mathrm{p}<0.05$, the comparison of "DM1" and "DM2".

amounting to $53.8 \pm 12.4 \mathrm{~s}^{-1}$ and $64.3 \pm 11.0 \mathrm{~s}^{-1}$, respectively ( $\mathrm{p}<0.01$, the comparison of DM1 and DM2). Thus, for the diabetes mellitus of both types the interaction of erythrocytes at first was accelerated and then abnormally decelerated, resulting in the appearance of the aggregates with increased stability.

\subsection{Effect of monafram on the processes of $R B C$ aggregation in patients with the type- 1 or type-2 diabetes mellitus}

Monafram had no visible effect on the indexes $t_{2}$ and A. Generally, in the normal state $(n=31)$ the only essential effect of monafam was the deceleration of the first stage of erythrocyte aggregation that manifested itself as the increase of the aggregation time $\mathrm{t} 1$ from $11.0 \pm 4.0 \mathrm{~s}$ to $12.9 \pm 5.3 \mathrm{~s}(\mathrm{p}<0.001)$. In the presence of the disease, monafram had an analogous effect. The background and the post-injection values of $\mathrm{t}_{1}$ amounted to $7.9 \pm 1.7 \mathrm{~s}$ and $8.3 \pm 2.0 \mathrm{~s}(\mathrm{p}<0.01)$ in the DM1 group $(\mathrm{n}=19)$, $6.5 \pm 1.3 \mathrm{~s}$ and $6.7 \pm 1.2 \mathrm{~s}$ in the DM2 group $(\mathrm{n}=12)$, respectively. The averaged indexes of the aggregate stability in all cases demonstrated only an insignificant decrease trend under the effect of monafram, although this trend was sufficiently greater expressed in the patients with DM2, in which the strength index for the smallest aggregates decreased from $60.2 \pm 14.9 \mathrm{~s}^{-1}$ to $49.9 \pm 14.1 \mathrm{~s}^{-1}(\mathrm{p}<0.07)$.

We divided each of the groups into subgroups in correspondence with the earlier developed criterion [19]: the indices of RBC aggregationand disaggregation being within $95 \%$ confidence interval for their normal values, which is a sign of normal aggregation $(\mathrm{Nn}, \mathrm{n}=$ 19; DM1n, $\mathrm{n}=8$; DM2n, $\mathrm{n}=5$ ), or beyond these limits, which is a sign of "hyperaggregation" $(\mathrm{Nh}, \mathrm{n}=12$; DM1h, $\mathrm{n}=11$; DM2h, $\mathrm{n}=7$ ). The analysis revealed that in the case of normal aggregation-disaggregation dynamics monafram still affected only $t_{1}$, increasing it in all subgroups by $5 \%-20 \%$. In the subgroups with "hyperaggregation" (see Table 3), monafram decelerated the erythrocytes aggregation ( $\left.\mathrm{t}_{1}\right)$ and, additionally, slightly reduced the stability of the aggregates $\left(I_{2.5}, \beta_{1}\right)$ both in the normal state and in the case of disease.
The data on the effect of monafram on the erythrocytes in healthy persons agreed with the analogous data obtained by us earlier. These data showed that the specific fibrinogen-RBC binding only slightly accelerated the cell interaction, which could facilitate the appearance of positive consequences of the normally reversible red blood cells aggregation. On the contrary, in the case of "hyperaggregation" (as a rule, transitory in the normal state) the specific interaction of fibrinogen with the RBC membrane could facilitate abnormal phenomena, related to the accelerated formation of cell aggregates having increased stability $[11,19]$.

In the case of the diabetes mellitus of both types, the influence of monafram on erythrocytes was similar to that in the normal state, which did not allow us to state a particular role of the specific mechanism in the RBC aggregation for this disease.

\subsection{Shear-induced RBC deformation in patients with type-1 or type-2 diabetes mellitus}

No possible abnormalities of the RBC deformation manifested themselves under the conditions of accelerated shear flow in the examined patients, suffering from DM of both types (see $\operatorname{tg} \alpha$, Table 4). Moreover, the increase of the deformation-beginning threshold was combined with greater cell stretching in the accelerating flow: the coefficient of correlation between $\dot{\gamma}_{b}$ and $\operatorname{tg} \alpha, \rho$ amounted to $0.48(\mathrm{p}<0.05)$ in the DM1 group and $0.67(\mathrm{p}<0.005)$ in the DM2 group. Analogous correlations were found in the normal group $\mathrm{N} 1(\rho=0.39, \mathrm{p}<0.001)$ and, to a greater degree, in the group N2 $(\rho=0.72, p<0.005)$. The increased index of the maximal erythrocyte stretching, unexpectedly discovered in the studied patients ( $\mathrm{ID}_{\max }$, Table 3), increased in the DM2 group in the process of the disease (the coefficient of the correlation between the disease duration and $\mathrm{ID}_{\max }, \rho$ amounted to $\left.0.55, \mathrm{p}<0.05\right)$. The increased index of cells maximal stretching, as well as the increased degree of their deformation in the accelerating flow in the case of increased deformation 
threshold indicated the resistance to the possible aggravation of hemorheological properties in the group of the examined patients.

The division of DM1 patients into 2 subgroups, in which $\dot{\gamma}_{b}$ was greater (DM1_1, $\mathrm{n}=6$ ) and smaller (DM1_2, n = 14) than the

upper boundary of the $95 \%$ confidence interval for its normal values (i.e., $\sim 23 \mathrm{~s}^{-1}$ ), and the utilization of data of the standard laboratory testing revealed that the subgroup DM1_1 differed from DM1_2 by narrower diameter distribution of erythrocytes $(\overline{11} .7 \pm 0.8 \%$ and $14.1 \pm 6.4 \%, \mathrm{p}<0.05$, respectively) and higher content of triglycerides in the blood plasma $(0.91 \pm 0.28 \mathrm{mmol} / \mathrm{l}$ and $0.66 \pm 0.14 \mathrm{mmol} / \mathrm{l}, \mathrm{p}<0.05$, respectively), which could partially explain the increased cell deformation threshold in the subgroup DM1_1. Generally, in spite of the factors fortunate for decreasing the erythrocyte deformability and the fact that in DM this decrease was reported in the literature $[4,20]$ the data obtained by us manifested the compensatory changes rather than the pathological ones at this level.

\section{Conclusion}

In patients suffering from type-1 or type-2 diabetes mellitus we found the accelerated first phase of erythrocytes aggregation, followed by the anomalously decelerated second phase of the process. In contrast to the normal state, in which the disintegration of the aggregates having different size frequently occurs with equal easiness, in the case of diseases, alongside with the general enhancement of the hydrodynamic strength of aggregates, the stability of the smallest ones grows particularly. The specific component of RBC aggregation mechanisms has no particular significance in the case of diabetes mellitus.

The deformation properties of erythrocytes in the case DM can change ambiguously and, alongside with the increase of the threshold effort required to initiate the deformation, the stretching of erythrocytes in the shear flow can increase, too.

The authors thank Prof. A.V. Mazurov (Russian Cardiologic Research and Production Complex, Moscow) for providing them with monafram and the staff members of the M.F. Vladimirsky Moscow Regional Research and Clinical Institute (MONIKI) for the execution of standard clinical and laboratory examination of the tested persons.

\section{Disclosures}

All authors declare that there is no conflict of interests in this paper.

\section{Acknowledgment}

The work was partially supported by the Russian Scientific Foundation, grant No. 14-15-00602. 